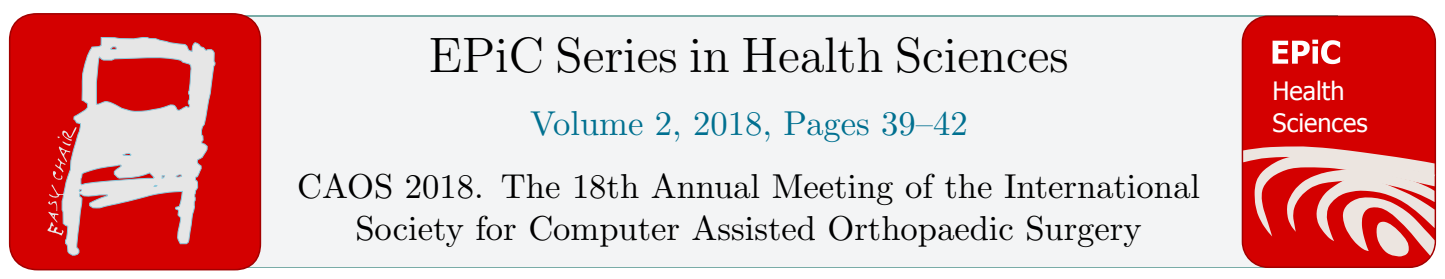

\title{
An Easy-to-use Portable Ultrasound based Device for the Measurement of the Pelvic Tilt
}

\author{
Guillaume Dardenne ${ }^{1,2}$, Jean-Philippe Pluchon ${ }^{1,2}$, Aziliz Guezou- \\ Philippe $^{1,2}$, Hoel Letissier ${ }^{1,2}$, Chafiaa Hamitouche ${ }^{1,4}$, Christian Lefèvre ${ }^{1,2,3}$, \\ and Eric Stindel $1^{1,2,3}$ \\ ${ }^{1}$ LaTIM, Inserm, UMR 1101, SFR IBSAM, Brest, 29200, France \\ ${ }^{2}$ CHU Brest, Brest, 29200, France \\ ${ }^{3}$ Université de Bretagne Occidentale, Brest, 29200, France \\ ${ }^{4}$ IMT-Atlantique, Dpt ITI, Brest, 29200, France \\ guillaume.dardenne@chu-brest.fr
}

\begin{abstract}
The orientation of the cup in Total Hip Arthroplasty plays a major role on the postoperative results. It has been considered for several decades that the cup should be oriented according to the safe zone defined by Lewinnek. However, this safe zone is not always suitable because of the inter-individual variability of the pelvic tilt during daily activities. We propose in this paper a non-invasive ultrasound based solution which can easily measure this patient specific parameter in order to thereafter otimise the cup orientation. The accuracy of this system was assessed with a specific pelvic phantom. A clinical pilot study was also performed on ten patients. The pelvic tilt was measured in three daily positions: the supine, sitting and standing positions. The average error was $1.15^{\circ} \pm 0.82^{\circ}$. The average pelvic tilt was $-97.1^{\circ} \pm 28.6^{\circ},-46.3^{\circ} \pm 12.8^{\circ}$ and $-9.0^{\circ} \pm 8.3^{\circ}$ for respectively the supine, the sitting and the standing positions. The high inter-individual variability of the pelvic tilt in different daily positions highlight the need to have a suitable device for the measurement of this patient specific parameter for THA. The proposed system is easy-to-use, portable and allows the pelvic tilt measurement in different positions without any additional x-ray radiation.
\end{abstract}

\section{Introduction}

The orientation of the cup in Total Hip Arthroplasty (THA) plays a major role on the implant stability, the hip function and the prosthetic wear (Scheerlinck, 2014). It has been considered for several decades that the cup should be oriented according to a safe zone (Lewinnek, Lewis, Tarr, Compere, \& Zimmerman, 1978). However, some authors mentioned that this safe zone is not always suitable because of the extreme inter-individual variability of the spine-hip kinematics which can 
significantly impact the pelvic behavior and thus the functional orientation of the cup during daily activities (Rivière et al., 2017).

Some solutions have been recently developed in order to take into account this patient specific parameter for the placement of the cup in THA (Pierrepont et al., 2016)(Lazennec et al., 2015). These solutions require either additional $x$-ray radiographs or a specific EOS $^{\circledR}$ imaging system. A first study in 2008 described the use of a localized ultrasound (US) probe to determine the pelvic tilt of a patient in different daily positions (Dardenne, Dusseau, Hamitouche, Lefèvre, \& Stindel, 2009). However, it requires the use of an expensive CAOS station. A similar concept has been more recently proposed which uses a laptop to acquire US images, a tablet to display information and a smartphone to localize markers (Kochman et al., 2017). However, it requires the presence of two physicians to (1) handle the US probe and (2) hold the smartphone for the localization of the markers.

A new easy-to-use portable device is proposed for the measurement of the patient specific pelvic tilt in different daily positions. The goal of this paper is to (1) evaluate the accuracy of this device and (2) show measurements performed on ten patients according to the standing, the supine and the sitting positions.

\section{Materials and Methods}

The pelvic tilt measurement device is composed of a L12-5L60N US probe from TELEMED ${ }^{\circledR}$ connected to a Microsoft Surface Pro ${ }^{\circledR}$ tablet having an embedded accelerometer (figure 1). The 3D localization of the US probe is determined with an ARUCO marker (Garrido-Jurado, Muñoz-Salinas, Madrid-Cuevas, \& Marín-Jiménez, 2014; Garrido-Jurado, Muñoz-Salinas, Madrid-Cuevas, \& Medina-Carnicer, 2016) attached to the probe and localized by the tablet front-facing camera. Both the camera and the US probe have been beforehand calibrated according to the approaches described in the PLUS open-source toolkit (Lasso et al., 2014). The Anterior Pelvic Plane (APP) is determined by acquiring both iliac spines and the pubic symphysis with the US probe. The pelvic tilt is determined by computing the angle between the APP and the vertical provided by the tablet accelerometer.

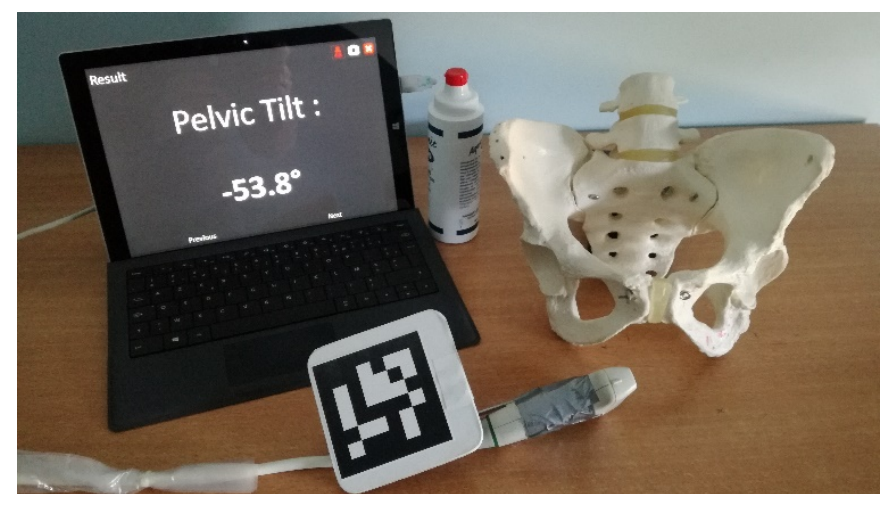

Figure 1: Pelvic tilt measurement device

The accuracy of the device was assessed with a pelvic phantom composed of a synthetic pelvis immersed in a box filled with water. The ground truth pelvic tilt value was provided by an inclinometer having an accuracy of $0.05^{\circ}$. The measurements were performed according to several tests including different pelvic tilts and different soft tissue thicknesses. The tilt was adjusted every $5^{\circ}$ 
between $20^{\circ}$ and $80^{\circ}$. For each tilt, the US probe was placed at 5,15 and $25 \mathrm{~mm}$ from the bony landmarks in order to simulate the soft tissue thickness. Twenty measurements were performed per tests. The error was defined as the angular difference between the pelvic tilt values provided by the US based system and the ground truth.

A clinical pilot study was also performed on ten patients requiring a THA. The pelvic tilt of each patient was measured in the supine, sitting and standing positions.

\section{Results}

In the figure 2, the middle line represents the median, the central rectangle spans from the first to the third quartile and the vertical line extends from the first to the ninth decile. Separated points represent values which are inferior or superior to respectively the first and the ninth deciles. Figure $2 \mathrm{a}$ shows the error of the device. The average error was $1.15 \pm 0.82^{\circ}$. Figure $2 \mathrm{~b}$ shows the pelvic tilt measurements performed on ten patients in the supine, sitting and standing positions. The average pelvic tilt was respectively $-97.1 \pm 28.6^{\circ},-46.3 \pm 12.8^{\circ}$ and $-9.0 \pm 8.3^{\circ}$. The inter-individual variability of the pelvic tilt was respectively $35.7^{\circ}, 48.9^{\circ}$ and $26.3^{\circ}$.

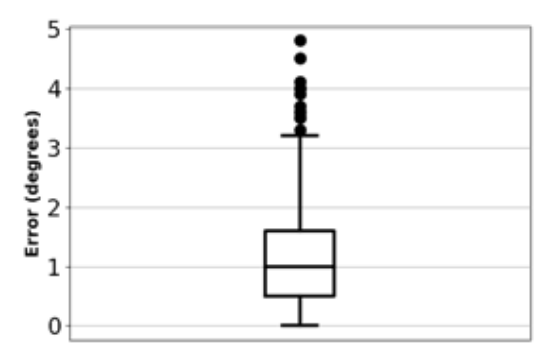

(a)

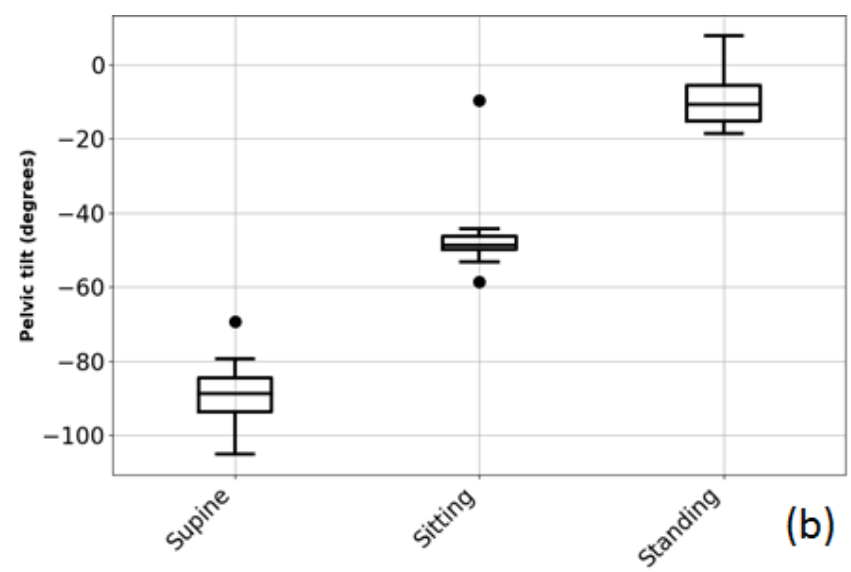

Figure 2: Error of the device (a) and pelvic tilt measurements on ten patients in the supine, sitting and standing positions

\section{Discussion}

The proposed system is easy-to-use, portable and allows the pelvic tilt measurement in different daily positions without any additional x-ray radiation (Pierrepont et al., 2016) (Lazennec et al., 2015).

As already highlighted by some authors (Rivière et al., 2017), the high inter-individual variability of the pelvic tilt confirm the need to have a patient specific approach for THA. Such a device for the pelvic tilt measurement is thus required. 


\section{References}

Dardenne, G., Dusseau, S., Hamitouche, C., Lefèvre, C., \& Stindel, E. (2009). Toward a Dynamic Approach of THA Planning Based on Ultrasound. Clinical Orthopaedics and Related Research, 467(4), 901-908. https://doi.org/10.1007/s11999-008-0408-z

Garrido-Jurado, S., Muñoz-Salinas, R., Madrid-Cuevas, F. J., \& Marín-Jiménez, M. J. (2014). Automatic generation and detection of highly reliable fiducial markers under occlusion. Pattern Recognition, 47(6), 2280-2292. https://doi.org/10.1016/J.PATCOG.2014.01.005

Garrido-Jurado, S., Muñoz-Salinas, R., Madrid-Cuevas, F. J., \& Medina-Carnicer, R. (2016). Generation of fiducial marker dictionaries using Mixed Integer Linear Programming. Pattern Recognition, 51, 481-491. https://doi.org/10.1016/J.PATCOG.2015.09.023

Kochman, A., Goral, A., Martin, T., Marek, W., Kozak, J., Morawska-Kochman, M., \& Synder, M. (2017). Application of Navigated Ultrasound for Assessment of the Anterior Pelvic Plane in Patients With Degenerative Hip Diseases. Journal of Ultrasound in Medicine, 36(7), 13731380. https://doi.org/10.7863/ultra.16.07016

Lasso, A., Heffter, T., Rankin, A., Pinter, C., Ungi, T., \& Fichtinger, G. (2014). PLUS: Open-Source Toolkit for Ultrasound-Guided Intervention Systems. IEEE Transactions on Biomedical Engineering, 61(10), 2527-2537. https://doi.org/10.1109/TBME.2014.2322864

Lazennec, J.-Y., Rousseau, M.-A., Brusson, A., Folinais, D., Amel, M., Clarke, I., \& Pour, A. E. (2015). Total Hip Prostheses in Standing, Sitting and Squatting Positions: An Overview of Our 8 Years Practice Using the EOS Imaging Technology. The Open Orthopaedics Journal, 9, 2644. https://doi.org/10.2174/1874325001509010026

Lewinnek, G. E., Lewis, J. L., Tarr, R., Compere, C. L., \& Zimmerman, J. R. (1978). Dislocations after total hip-replacement arthroplasties. The Journal of Bone \&amp, 60(2), 217-220. Retrieved from https://insights.ovid.com/crossref?an=00004623-197860020-00014

Pierrepont, J. W., Stambouzou, C. Z., Miles, B. P., O'Connor, P. B., Walter, L., Ellis, A., ... Marel, E. (2016). Patient-specific Component Alignment in Total Hip Arthroplasty. Reconstructive Review, 6(4). Retrieved from https://www.reconstructivereview.org/ojs/index.php/rr/article/view/148/202

Rivière, C., Lazennec, J.-Y., Van Der Straeten, C., Auvinet, E., Cobb, J., \& Muirhead-Allwood, S. (2017). The influence of spine-hip relations on total hip replacement: A systematic review. Orthopaedics \& Traumatology: Surgery \& Research, 103(4), 559-568. https://doi.org/10.1016/j.otsr.2017.02.014

Scheerlinck, T. (2014). Cup positioning in total hip arthroplasty. Acta Orthopaedica Belgica, 80(3), 336-47. Retrieved from http://www.ncbi.nlm.nih.gov/pubmed/26280607 